\title{
YI.
}

\section{Ein Beitrag zur Mechanik der Beinprothese.}

Von

\author{
Eriedrich Wilhelm Brekenfeld, \\ Künigl. Assistenzar $九 t$.
}

Mit $t^{2}$ Abbildungen.

Die Frage nach der Herstellung einer Prothese für ein eingebüsstes Glied, die nach jeder Richtnng hin den an sie gestellten Anforderungen gerecht werden soll, ist mehr denn je in dem gigantischen Völkerkrieg 1914:15 akut geworden.

Es ist nicht nur die Mechanil, von del Ausserordentliches verlangt wird, weil sie Ausserordentliches zu leisten imstande ist, sondern ein jeder verstümmelte deutsche Soldat ist dank seiner verhältnismässig hohen Bildung, dienk der Kulturhöhe des deutschen Volkes sittlich und geistig stark genug. um auch mit einem künstlichen Gliede auf jeden Fall den Tersuch za machen. den Kampf ums Dasein aufzunehmen.

Doch nicht nur dieser wichtige Faktor allein, die Sehnsucht nach der alten Arbeit und der Abschen vor dem Müssiggang und der Bettelei, wird uns vor der Flut von kriegsinvaliden Leierkastenmännern und Almosenempfängern bewa!lren, wie sie nach den Freibeitskriegen und dem deutsch-tranzösischen Feldzug 1870/71 aufgetreten ist, sondern auch die staatliche Fürsorge wird einen jeden anhalten, diejenige Arbeit zn leisten, die nach den bisherigen Erfahrungen der orthopädischen Chimrgie gelejstet werden han, einesteils, um die riesige Summe der Kriegsversorgungsgelder auf ein Winimum zu bringen, anderenteils aber auch zum Jutzen und Frommen des betreitenden Lriegers.

Das Bestreben, ein verlorenes Bein durch ein liinstliches zu ersetzen. ist nachweislich rom frühen Mittelalter an bei allen zivilisierten Völkern lebendig gewesen. Das hölzerne Stelzbein suchte sehr bald die Krücke zu verdrängen und ermöglichte oft ein leidlich gutes Lasein, da es die Arme beim Gehen und Stehen zur Tätigkeit frei liess.

Schon sehr früh ging man dann dazu über, das künstliche Glied mehr der Natur vachzubilden und schui mehr oder weniger komplizierte Apparate, wie es beispielsweise der eiserne Arm Götz von Berlichingens zeigrt. Bis zum heutigen Tage haben die einfachsten Holzglieder neben den sinnreichsten Konstruktionen bestanden. Es ist dies ein Zeichen dafür, dass jerles von beiden seine grossen Vorzüge und Nachteile besass, ganz abgesehen davon, dass sich die meisten mit dem billigsten Ersatz begnügen mussten. Ein treffliches Beispiel hierfür führt $\mathrm{K}$ arpinski in seinem hochinteressanten 
Werk „Studien über künstiche Gilieder“. das er 1981 mit einem lehrreichen Billeratlas im Anftage, dts kïniglich prenssischen liregsministeriums herausgegeben hat, an. Er zitiert dort auf Seite 118 den Franzosen Renault. der in einem Bericht über die Branchbarkeit einer rom eirem Studenten der Medizin namens Richard Cantegril konstruierten komplizierteren Beinprothese diese unter dem Hinweis auf die vorzügliche Branchbarkeit des Holystelzbeines ror der Akademic der Medizin in Paris mit folgender Begründung verwirft: .General Caffarelli, welcher bei der Nord-Armee im ersten Revolutionsliriege ein Bein verloren, habe nach vergeblichen Versuchen mit mehreren lïnstlichen Füssen sich endlich genötigt gesehen, zu dem gewöhnlichen hölzernen Bein zuriackzukehren, das er dann auch in İypten und bei der lielagerung von saint Jean diAcre getragen, wo ein glorreicher l'od seine Laufbahn beendigt: auch sei so in der letzten Zeit der Held von Vincennes, General Daumesnil, nacio unter den Angen der Akademie angestellten vergeblichen Versuchen mit künstlichen Füssen, ebenlalls wieder zu lem einfachen hölzernen Beine zurückgekehrt, mit welchem ar tïglich in dem Parke ron Vincennes jage."

Anch ein so bedentender (hirurge, wie Bardeloben, betrachtete die komplizi rteren kïnstlichen Glieder mit nicht geringer skepsis: "Es bedarf keinel grossen Cberlegung. um einzusehen, dass der liumstreiche Bau der Glieder von dem geschicktesten Mechanikus doch nur sehr düritig nachgeahmt werden kann und dass namentlich die aktiven bewegungsorgane sich so gut wie gar nicht ersetzen lassen . . . . . Dazu sind sehr komplizicrte Einrichtungen erforderlich, die einen sehr hohen Prtis und eine geringe: Dauerhaftigkeit bedingen...... Im allgemeinen werden wir solche Apparate bevorzugen müssen, die möglichst einfach. leicht und dauerhaft sind . . . . Zum Stelafuss kehren auch diejenigen, welche sich mit einer kostspieligen künstlichen Extremität verseben haben, ganz gewöhlich zurück, wenn sie grössere Mïrsche machen oder indere Anstrengungen unternehmen wollen, weil jene künstlichen Cilieder auf die Daner zu schwer sind. Wit dem stelzfuss aber lemen die meisten gewandt die verschiedenartigsten Bewegungen auszufïhren (selbst "Tanzen. Springen und Reiten)"; das sind sätze ans Bardelebens Lehrbuch der Cbiruricie.

Im meisten liopfzerbrechen hat den unermidllichen .Mechanilern bei der Herstellung einer müglichst naturgetreuen Beimprotlese von jeber das Fussgelenk gemacht. Vun len einfachen federnden Gummistiefeln und den Stahlfederkonstruktionen, den primitivsten Scharniergelenken und den schon sinnreicheren Kugelgelenken bis zu dem sehr kunstrollen Stahllager-Schaukelplattengelenk von Dürflinger ist wohl kaum ein System unversucht geblieben.

Es ist das Verdienst der allerjüngsten Zeit, insonderheit Hoeftmans, durch rastlose, scharie Beobachtung mit praktischem Blick eine Beinprothese konstruiert zu haben, die dem Träger das Sicherheitsgefühl wie ein Stelzbein, das moralische Bewusstsein, ein möglichst naturgetrenes Bein zu hahen, wie es die übertrieben komplizierten Fussgelenkkonstruktionen anstrebten, verschafft. So gut es gelungen war, ein brauchbares Kniegelenk herzustellen, 
so schlecht wollte es glücken, dem Fuss in vorteilhafter Weise die Eigenschaft des Federns zu verleihen.

Alle bisherigen Konstruktionen, bei denen sich der Fuss gregen den künstlichen Lnterschenkel bewegen liess, hatten den Nachteil. dass der Trïger diese Fussbewegungen nicht in seint: Gewalt hatte, gan? abresehen davon, dass die difizile Mechanik meistens Teile anfwies, die bald und leicht zerbrachen, oder geölt und gereinigt werden mussten, oder sich abnutaten und stark unter fenchten Witterungsverhälmissen zu leiclen hatten. Hoeftman hat, dank seiner reichen Erfahrung auf diesem Gebiete immer wieder Ferbesserungen auf Verbesserungen vornehmend, herausgefunden, dass das Gefühl der Sicherheit und damit der Crad der Arbeitsleistung wïcust, je steifer das Fussgelenk gearbeitet ist, und somit sein hörhstes Iass erreicht, wenn der Unterschenkel fest in den Fuss ubergeht. Fon anderer Seite war zunächst schon die Konstruktion verlassen, die eine allseitige Lewegung des Fusses ermöglichte und war zu einem Gelenk übergegangen worden, das nur eine Bengung und Streckung zuliess. Die Mechanik war sehr einfach. Der Gelenkanteil des linterschenkels stand mit seiner Konvexität der dazu parsenden lionkavität les Fussanteils am Gelenk gegenüber. Beide wurden in einer derartigen Fintfernung durch zwei seitliche Stahlschienen mit Scharniergelenken voneinander gehalten, dass eine ausreichende Benge- und Streckbewegung des Fussklotzes möglich war. For und hinter der Fussgolenkachse wurde nun das Fussgelenk dadurch vervollstïnligt, dass zwischen Unterschenkel und Fuss je eine derbe spiralfeder angebracht war. Te nach der Belastung des Fusses wurde die eine oder die andere Feder zusammengepresst. Es zeigte sich bei dieser Konstruktion bald der grosse Xachteil, dass eine der beiden Federn oft sprang und Reparaturen nötig waren; besonders geschah dieses naturgemïss wegen der starkeren Belastung bei der vorderen spirale, obwohl man sie deswegen schon stälier arbeitete als die hintere.

Hoeftman benutzte deshalb von vornherein, wie ts auch PristerBerlin getan hat. statt der Federn elastische Ciummipufter. Aber anch diese erwiesen sich keineswegs als dauerhaft. Sie nutzten sich sehr schnell ab und erfullten so baid nicht mehr ihren tsweck.

Ein grosser Vacisteil aller dieser Konstruktionen bestand noch in folgendem: Ging der betreffende Prothesenträger beispielsweise eine Treppe hinauf und achtete nicht genan darauf, dass er den ganzen Fuss auf die nächste höhere Stufe setzte, geschah dieses nur mit dem Sohlenteil, so rutschte er bei stïrkerer Belastung dadurch, dass er den Fuss nicht direkt in seiner Gewalt hatte, ab und wiurde gegen seine Prothese misstrauisch. Es ist dieses ein P'unkt, auf den nicht genug hingewiesen werden kann. Das Hinauf- und Heruntergehen der Treppe ist mit die erste Cbung, welche der Betreffende mit seiner Prothese auszufiiluren hat. Erleidet er aher schon dabei Missgeschick und bekommt ein Gefühl der. Lnsicherheit, so hat er rom ersten Hale an zu seinem künstlichen Jein kcin rechtes \%atrauen. Anch beim Gehen anf unebenem Gelïnde, bei Garten- und Feldarbeit wird ein 
bewegliches Fussgelenk oft zu einem Abgteiten führen und dem lrïger dils nütige iretühl, fest auf seinem künstlichen Fuss zu stehen. nehmen.

Ansserordentlich ins fewicht fällt dieser Naciteil zun Beispiel anch bei vielen sportlichen letätgungen, wie Reiten und Radeln, wo anf dem vorderen Teil des Fusses die Hauptkiaft ausgeübt wird. Den lieiter oder Radler wird dabei stets zum Bewusstsein kommen. dass er nicht Herr seiner Fussbewegungen ist.

Alle diese liedenken laben Hooftman dazu grefuhrt. eine Beinprothese mit völig steifem Fussgelenk zu konsiruieren, die nebenher dusch ihre ausserordentliche Einfachbeit, Leichtigkeit nnd Billigkeit ron nnschätzbarem Forteil ist. Tas einiache Modell eines deartigen Verbandes zeigt Fig. 1.

Von ler Interschenkelhülse gehen zwei

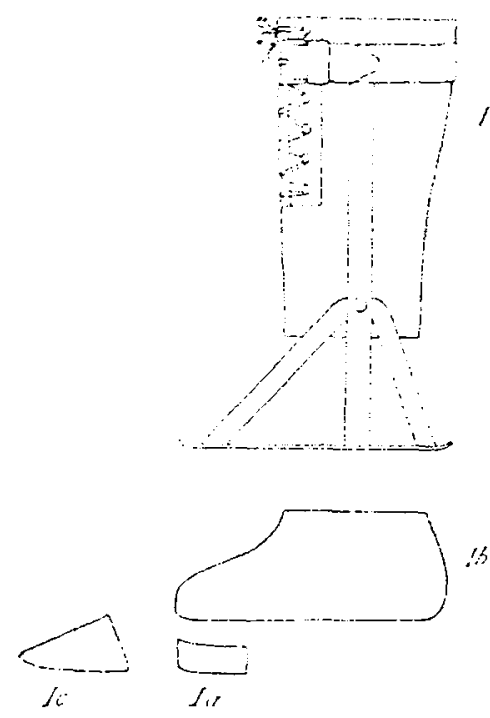

Eig 1. seitliche Schienen hinab zu einer stiahlenen Fussplatte, die so gut wie ganz gerade. nur an den Fnden rorn und hinten leicht in die Höhe grebogen ist, damit bein Gehen der Fuss etwas besser angewickelt werden kinn. Zur grösseren Stabilität wird rorn und hinten eine Strebe nach den Seitenschienen gemacht (vgl. Fig. 1: Die kranken grehen einfach auf dieser l'atte und rwar gut und tadellos. Will nan sie nun noch einigermassen gut aussehend machen, so wird dieser Apparat in einen gewöhnlichen Stiefel Fig. 2), der natürlich keinen zu hohen dbsatz hiben muss. hineingesteckt. Damit der Absat: nicht den Gehapparat nach rorn überkippen macht, wird vorn ein kleiner keil hinzugefügt, der der Höhe des Absatzes entspricht (Fig. 1a). Um ein Zusammentallen des Leders zu verhiiten, wird einfach ein Holzkcil (Fig, 1 b) von etwa Fussfasson zwischen die schienen gelegt and vorn in den Stiefel irgend ein Stiick Fila oder etwas Rosshaar hineinpraktiziert. um auch dort ein Zusammenfallen zu rermeilen (lig. 1 c). Bei dieser Anordnung bekommt der Patient, indem die steife Soble etwas federt, einen guten natirlichen Gang. so dass man den Leuten gar nicht ansieht, dass ihnen ein Fuss fehlt.

I)ie Befestigung der Beinprothese wird sich natürlich nach dem vorhandenen stumpf zu richten haben. Handelt es sich um eine Amputation im unteren Irittel, ja selbst bis zur Witte des Cnterschenkels, so genügt ein Hülsenapparat, der nur genau den Lnterschenkel umschliesst und seinen Stützpunkt in der Höhe der Tuberositas tibiae bat. \%ur Sicherheit, damit nicht ein Alygleiten von dem immerhin konischen Unterschenkel vorkommt, wird diese Unterschenkelbülse mit zwei seitlichen Riemen gehalten, die an einer zirkulïren Manschette oberhalb des Kniegelenks befestigt sind. Ist die Amputation sehr hoch in Linterschenkel ausgeführt, so dass in Anssicht 
genommen war, den Kranken mit gebeugtem Kniggelenk gehen zn lassen, so genügt doch selbst ein so kurzer Stumpf, um das Gehen mit gestrecktem Cnterschenkel und beweglichem hiniegelenk zu ermöglicinen. lis muss dann noch eine Schienenverbindung und eine Hülse für den Oberschenkel hinzugefügt werden, um so die Last zam 'Teil auf den Oberschenkel zu übertragen. Die Knicgelenkschienen sind selbstrerstïndlich beweglich. Ganz ähnlich ist die Kniegelenkkonstruktion bei Amputation in Oberschenkel. Twei im hiniegelenk bewegliche Schienen verbinden Ober- und Lnterschenkelhüise. Eine der Kniewölbung entsprechende nach unten spitz zulautende Lederkappe schliesst sich rorne so an die Oberschenkelhülse an, dass sie bei gestrecktem Bein bis etwa zur Hälfte in die Enterscbenkelhülse hineinragt. Sie bewirlt dadurch ein grleichmässiges Gleiten beim Bewegen des Cinterschenkels gegen den oberschenkel und verhindert, dass sich die llose des l'üger's in das Kniegelenk einklemmt und denselben dadurch vielleicht zu Fall bringt. Durch cin starkes Gummiband. dis vorne ron oberen Teile der Oberschenkelhülse zur Unterschenkelhülse herabläuft und daselbst, durch entsprechende einfache Forrichtung straffer oder lockerer gespannt werden kann, ist noch eine Regelung des Krümmungsgrades des liniegelenkes nöglich. Es ist bei der Prothese für Oberschenkelstumpt gan\% besonders darauf zu achten, dass der Apparat sich genau auf den Schambein-Sitzbeinring stützt. Te besser er nach dieser lichtung hin gearbeitet ist, um so fester wird er am Stumpt sitzen und um so vollkommener seinen $\%$ weck erfïllen. Die bekannten 'l'rag- und Haltegurte vervollständigen die P'rothese.

Es kommt nun verhältuismüssig bäutig vor, dass der Oberschenkelstumpf ausserordentlich fettreich. Heischig ist, dass die Weichteile an dem Knochergerüst keinen genügenden Halt mehr haben, vielmebr schwammig-lasch willkürlich hin- und herpendeln. Ein solcher Stumpt bietet der Protiese

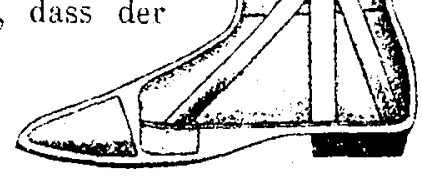

Fig. 2. natürlich einen sehr geringen Halt, so dass er ein Abrutschen begünstigt und der Hülse gestattet, sich frei gegen die Oberschenkelachse zu bewegen, sowie der Patient das künstliche Bein in irgend einer Weise belastet. Es ist dieses selbstverständlich um so mehr der Fall, je kürzer der Stumpt ist.

Man kann diesen recht erleblichen Nachteil auf eine sehr einfache Art korrigieren; man muss, um hier ein möglicbst festes Haften der Prothese an dem fleischigen, kurzen Stumpf zu erzielen, das Nodell derartig abnehwen, dass die gesamten Weichteile ein wenig in O-Stellung gedrïngt werden.

Als sprechendes Beispiel für den grossen Wert des steifen Fussgelenks bei Beinprothesen möchte ich nun ein trefliches Beispiel ans der allerjüngsten 7eit anführen. Es stellt nicht nur nnserer heligen Technik das beste Zeugnis aus und zeigt nicht aur den gewaltigen Fortschritt, den die orthopädische Chirurgie, die vor einem Menschenalter noch in den Kinderschuben steckte, gemacht hat aufs klarste, sondern es ist auch dazu angetan, gerade in dieser 7eit der massenhaften Verstümmelungen die Betroffenen, die ihr Glied opfer- 
frendig für Dentschlands Lhre hingegeben haben, for eine 'latsache zu stellen, die die l.ente mit einer unschätzbaren \%uversicht für die Znkunft erfüllt. Es handelt sich in diesem Falle um einen Hauptmann, der am $i$. Sep-

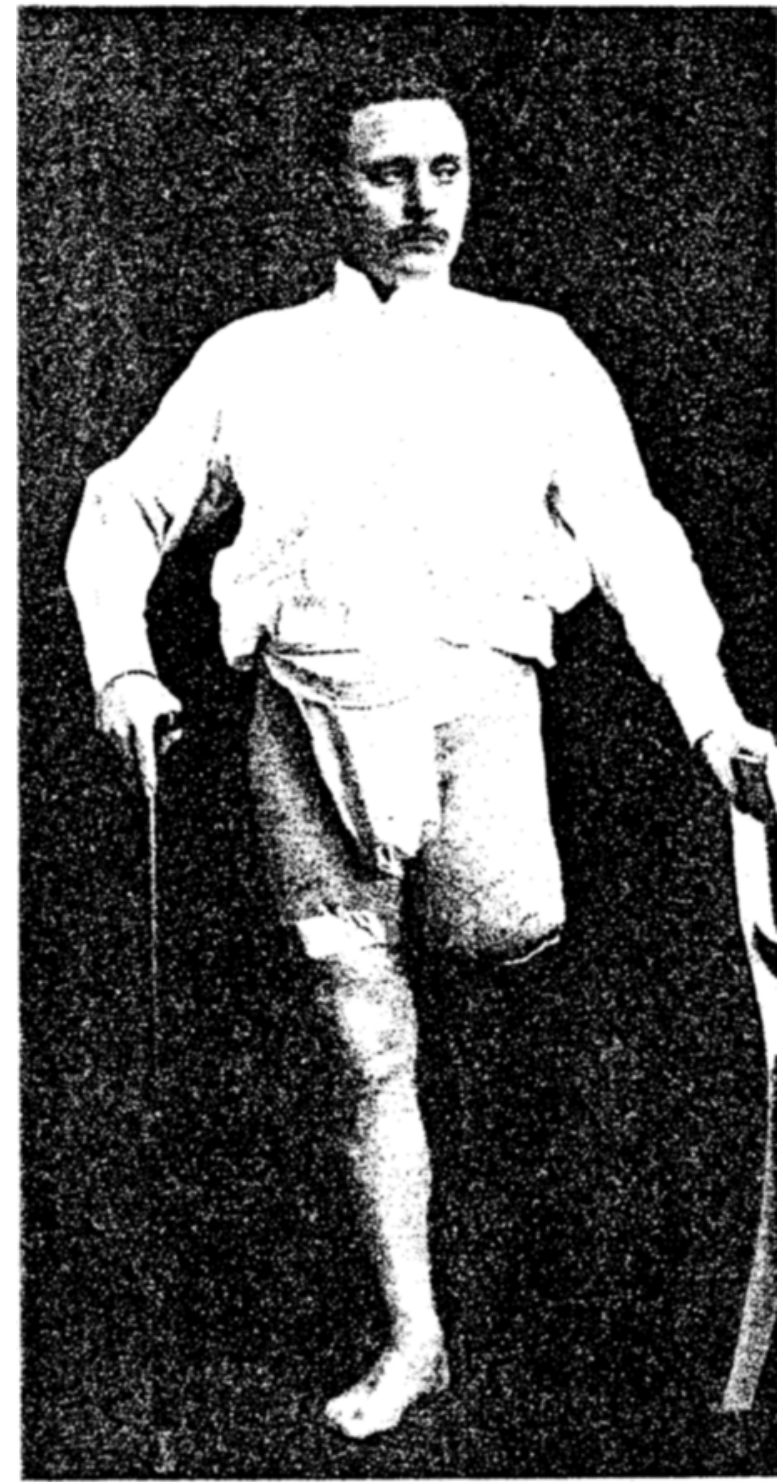

Hig. 3.

tember 1914 bei Vitry le Fruncois durch einen Granatschuss im linken Kniegelenk schwer verwundet wurde.

Er wurde am 28. November 1914 in die Krijpelbeil- und Jehransialt (Hindenburghaus! za Lönigsber i Pr. aufgenommen, Dik eine Erhaltung des 
ganzen Beines ausgeschlossen war, so hatte man bereits in Bonn am 6. (1)tober 1914 eine Amputation in der Nitte des linken Oberschenkels ansgeführt. Bis aut eine kleine Ecke an ler Aussenseite der Narbe war der

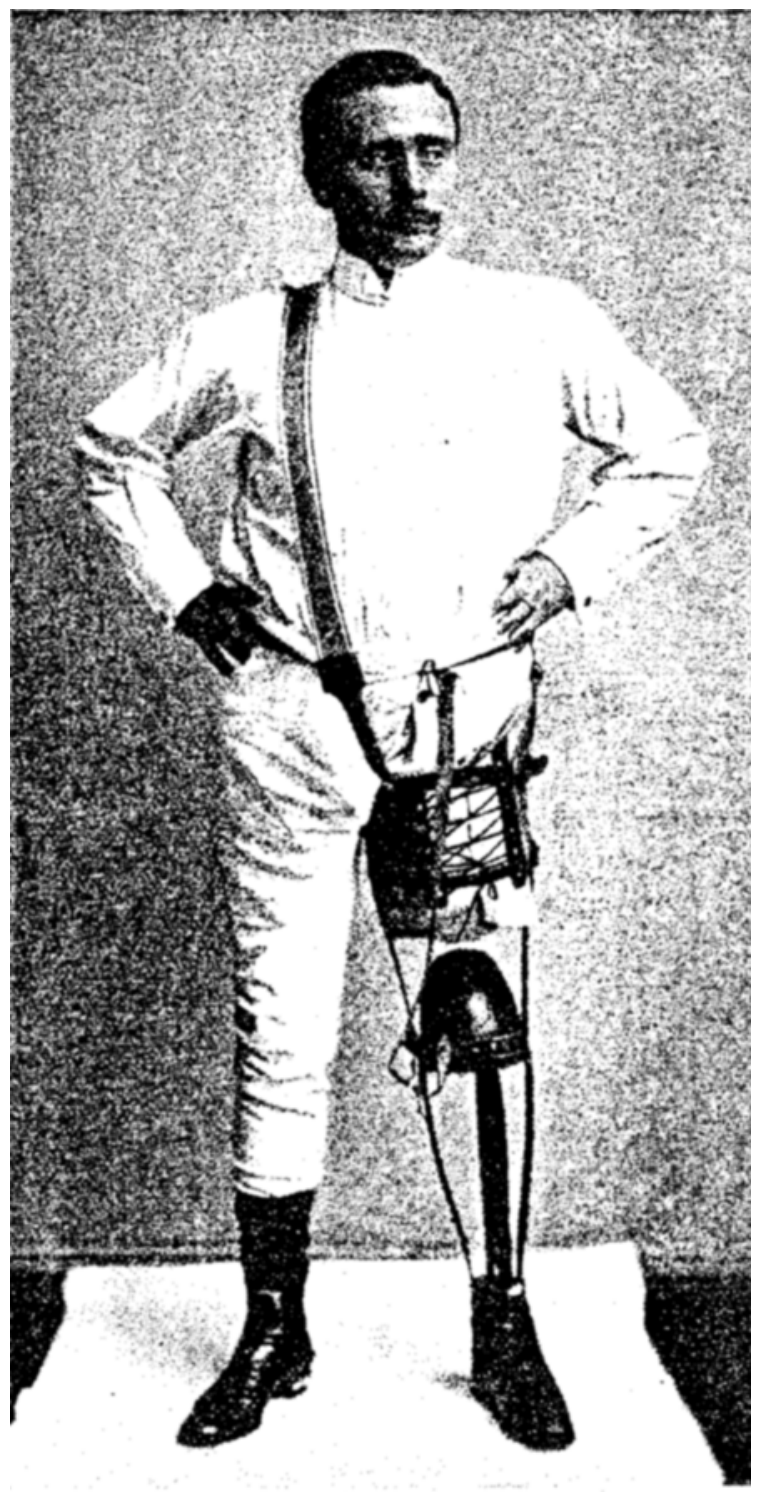

Fig. 1.

Stumpf gut verheilt. Er war in Hüftgelenk gut beweglich, aber insofern sebr ungïnstig, als er stark geschwollen war (s. Fig. 3).

Um ein möglichst festes Haften der P'rothese an dem kurzen, schwammigen stumpf zu erreichen, wurde die oben erwähnte Methode in Anwendung ge- 
bracht, die Weichteile wurden beim Abnehmen des Modells in O-Stellung gedrängt. Es lonnte so die Prothese weder abgleiten, noch war besonders bei Adduktionsbewegungen ein allzu starkes Kreuzen der Beinachse mit dem Stumpfe möglich. Der sehr günstige Erfolg dieser kleinen, bequemen Hassnahne erhellt schon daraus, dass man an die Prothese gegen 20) Pfund anhä̈ngen kimnte, ohne diss dieselbe heruntergerissen wurde. Sie bestand zuerst ans Filz wie alle die gebräuchlichen Übungsprothesen, mit der üblichen Verlegung des Kniegelenkes nach hinten, wie es Hoeftman in seinem Aufsatz "Prothesen fiir untere Extremitaten" eingehend beschrieben hat.

Am 30. November 1914 machte der Patient den ersten Gehversuch (s. Fig. 4). Er konnte sofort ziemlich sicher gehen. lir wurde dann gleich angelalten, die Treppe hinauf- und herunterzugehen. Es ist hierbei sehr wichtig, mit dem Treppanfwärtssteigen anzufingen. Das Hinabsteigen anfänglich natiirlich mit tiner gewissen Lnsicherheit ansgeführt, erweckt bei dem l'atienten zuerst stets den Gedanken, er liönnte herunterfallen, wird dadurch leicht schwindlig und bekommt von vornherein eine Abneigung gegen die Stufen. Sieht er aber, dass das Hinanfsteigen leidlich gut und sicher geht, so hat er sofort grosses Zutrauen zu seinem küinstlichen Bein und lässt es sich durch das [nsicherheitsotühl beim ersten Heruntersteigen nicht verdriessen. Cnserem Patienten machten die paar Stufen selbst beim ersten Male keine grossen Schwierigkciten, und es währte nur ganz lsurze Zeit, da war ihm keine Treppe der Klinik mehr ein Hinlernis.

Cm die Bewegungen des Stumpfes im Hüftgelenk ausgiebig zu machen und die gebliebene Muskuiatur zu kräftigen, wurde am 9. Dezember 1914 nit den Übungen im Zanderinstitut begonnen. Da es der sehnlichste Wunsch des Patienten war, es mit seinem künstlichen Bein mindestens so weit zu bringen. um wieder reiten zu künnen, wurde ein Versuch hierzı gleich beim ersten Mitle auf dem Reitapparat gemacht, wobei er denselben natülich entgegen der Gewohnheit von der rechten Seite her besteigen musste; das Herühersiuwenken des linken künstlichen Beines wurde gleich beim ersten Vale mit einer sehr erfreulichen, viel rersprechenden sicherheit ansgefïhrt.

Schon am 1t. Dezember 1914, also nur o Tage nach dem ersten Reitrersuch im Zandersaal, begann der Hanptmann seine Reitiibungen auf einem ruhigen P'ferd in der Bahn (s. Fig. à und 6).

Das Aufsteigen von einem Schemel aus ging ansgezeichnet, der sitz liess nichts zu wünschen übrig. Der Reiter fühlte sich so sicher, dass er schon am nüchsten Tage Gulopprersuche machte. Er setzte diese Utbungen von der Hoeftman'schen Klinik atus noch bis zum 22. Dezember 1914 fort und brachte es mit seiner Prothese zu einer derartigen Vollendung, dass er am 23. Dezember 1914 die Anstalt verlassen konnte und sich am 28. Dezember 1914 wieder seinem Truppenteil zur Dienstleistung zur Verfügung stellte.

Beispiellos ist nun, was uns der Hauptmann, mit dem Hoeftman in stindigem Schriftwechsel geblieben ist, über seinen Dienst und seine Leistungen schreibt. 
Beim Ersatzabataillon in Koblenz tat er zunächst Garnisondienst und watr für gewöbnlich von $8 \mathrm{l}$ hr vormittags bis 2 thr nachmittags uni von + Lihr bis spät in die lacht tiitir. und zwar hamptsichlich auf den Beinen

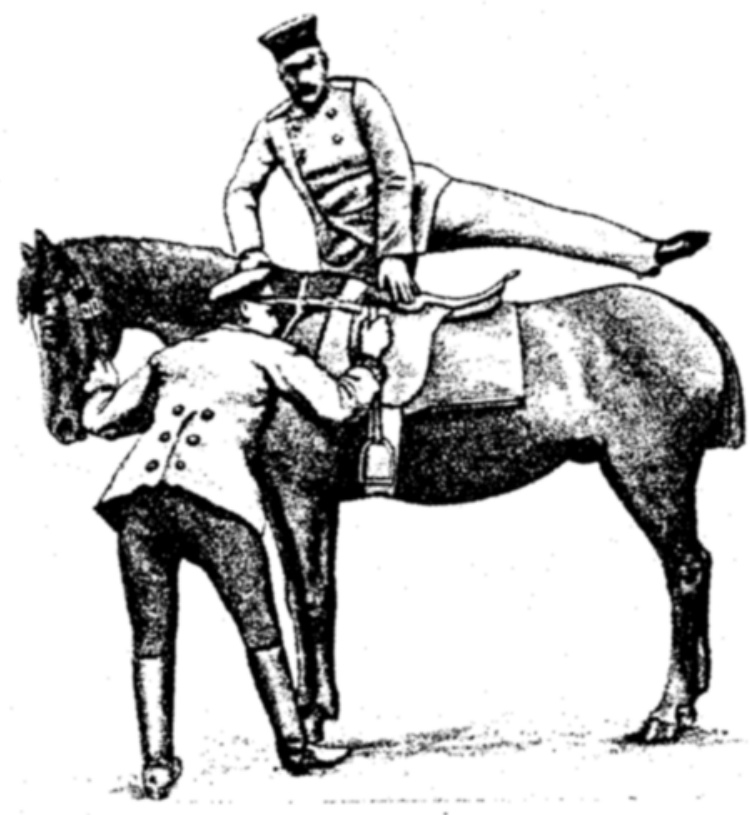

Fis. 5.

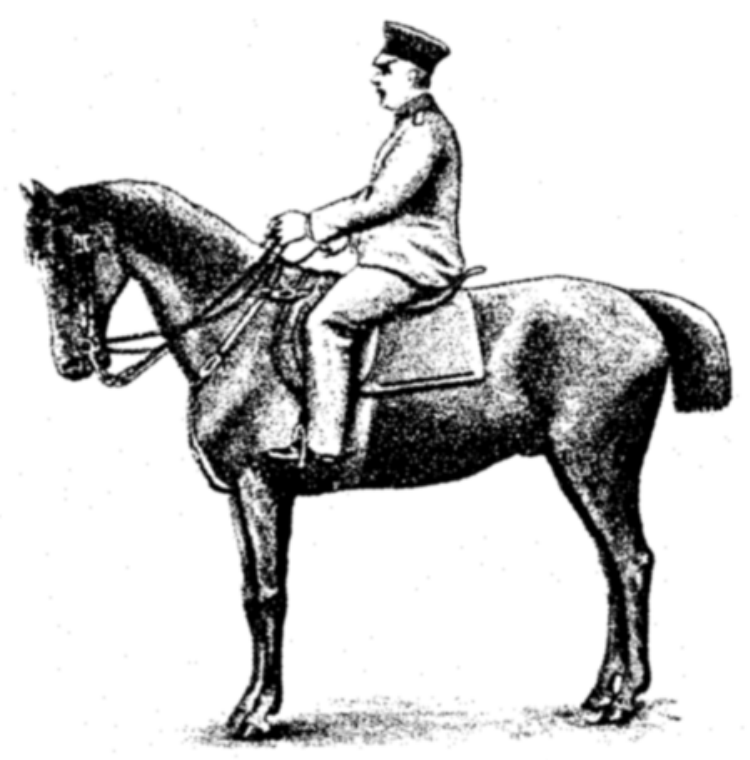

Fig 6. 
was besonders hervorgehoren werden muss, da das Gehen und Stehen die Patienten mit künstlichem bein anfünglich am meisten anstrengt.

In einem seiner ersten briefe hebt der Hatuptuann besonters herror. dass er an einem Tage a Stunden hintereinander ohne jegliche laast gegangen sei, nur mit einem gewölnlichen Stock in der Hand.

Endele Februar etwa wurde er dann nach Vanum beordent zum bienst beim Generaliommando. Finige Tigge später wurde sein sehnsüchtigster Wunsch erfiillt: Höheren ortes betrachtete man ihn wieder als felddienstfahig und trilte ihn, wie er in einem Briefe rom 1. Wärz 1915 schreibt, dem Nachrichtendienst fom Grossen Hauptquartier zur Front zu.

Dieses darf wohl als Gipfelpunkt alles dessen angesehen werden, was je: mit einem kïnstlichen bein geleistet worden ist. Es ist bekannt, dass die Ofliziere rom Nachrichtendienst, sehr oft nicht an ihr lagesuluantum Schlaf denken können. lass sie zumeist den ganzen lag; die ganze lacht. unterwers sind, sei es zu luss, zu Pferde oder zu Kraftwagen. sei es. dass sie lange stehen. eilig gehen oder Treppen steigen müssin, festen Boden, Ackershlollen oder Morast unter iuren Füssen baben, es werden an die heinprothese in diesem Falle genau dieselhen hohen Anforderungen gestellt, wie an das bein jeden gesunden Ordonnanzoftiziers. Dieser Hauptmann liefert das beste Zengnis tür deu Wert der ohen angefuhrten Hoeftman schen Beinkonstruliom. vor allem des steifen Eussgelenkes; er erbringt aber auch den ungemein wichtion Bewris. Was der Mensch erreichen kann. wenn er den Willen und die Kraft hat. nicht Krïppel za bleiben. Es eriftinet dieser Fall sehr frendige und sehe wichtige Anshlicke in die \%uhunft der hilipgelliursorge in einer \%eit, wo Viiker wider Völker steher und sich regeneitig verstimmein. Ich sage vielleicht nicht zu viel, wenn ich behaupte. dass wir mit einer solehen Errungenschaft. wie es die Konstrulation dieser modernen beinprothese ist, an einem Wendepankt der sozialen lirippelfiirsorge angelangt. sint.

lch schliesse meine Ausfiihrung mit einer Janksagung an Herrn Geweimrat Professur Wr. Hoeft man, der mir bereitwillig sein Waterial zar Verfigung gestellt bat.

\section{Literatur.}

Gocht, hiustlirhe Gleder, ein beitrag zar mechanischen und orthopädischen chirargie.

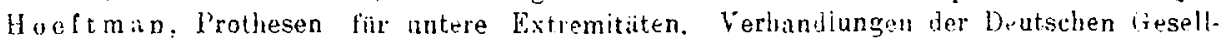
schaft für orthopädische Chirurrie. Bu. Vl.

Dorselbe, Prothesn als Ersatz fin veriorene Kxtremititen. Ferhandungen der Dentschen Gesellschaft für ortbopädiscbe Chirurgie. Bi. VIII.

Derselbe, Wiederberstellung der Arbeitsfahigkeit durch Prothesen. Zeitschrift t. Orthop. Bi. Xxxill.

Karpinski, Studien übər künstliche Glieder. 